Viharos, Zs. J.; Kis K. B.: Survey on Neuro-Fuzzy Systems and their Applications in Technical

Diagnostics and Measurement, Measurement, Vol. 67., 2015., pp. 126-136., (doi: http://

dx.doi.org/10.1016/j.measurement.2015.02.001), SCI, Impact Factor: 1.526.

\title{
Survey on Neuro-Fuzzy Systems and their Applications in Technical Diagnostics and Measurement
}

\author{
Dr. Zs. J. Viharos ${ }^{*}$, K. B. Kis
}

The Computer and Automation Research Institute, Hungarian Academy of Sciences, Budapest, H-1111 Hungary Tel.: +36 12796 195, E-mail address: \{viharos.zsolt ${ }^{*}$, kis.krisztian\}@sztaki.mta.hu

\begin{abstract}
Both fuzzy logic, as the basis of many inference systems, and Neural Networks, as a powerful computational model for classification and estimation, have been used in many application fields since their birth. These two techniques are somewhat supplementary to each other in a way that what one is lacking of the other can provide. This led to the creation of Neuro-Fuzzy systems which utilize fuzzy logic to construct a complex model by extending the capabilities of Artificial Neural Networks. Generally speaking all type of systems that integrate these two techniques can be called Neuro-Fuzzy systems. Key feature of these systems is that they use inputoutput patterns to adjust the fuzzy sets and rules inside the model. The paper reviews the principles of a NeuroFuzzy system and the key methods presented in this field, furthermore provides survey on their applications for technical diagnostics and measurement.
\end{abstract}

Keywords: Technical diagnostics, Neuro-Fuzzy systems, Measurement

\section{Introduction}

As two important techniques of artificial intelligence, Fuzzy Systems (FS) and Artificial Neural Networks (ANNs) have many applications in various fields such as production, control systems, diagnostic, supervision, etc. They evolved and improved throughout the years to adapt arising needs and technological advancements. As ANNs and Fuzzy Systems had been often applied together the concept of a fusion between them started to take shape. Neuro-Fuzzy systems were born which utilize the advantages of both techniques: they have learning and generalization capabilities and at the same time they reveal the functionality stored in the model. To reach this behaviour they are able to learn and tune their parameters based on inputoutput patterns (learning phase) and then they work like a fuzzy logic system (execution phase), too. These combined features make this type of systems useful when solving complex problems also for technical diagnostic and measurement assignments. The paper contains seven sections. After the introduction the second section presents NeuroFuzzy applications of the last two decades in technical diagnostics and measurement. The third section describes the two main components of a Neuro-Fuzzy system followed by the forth one reviewing the progression of the Neuro-Fuzzy systems and the modern solutions used today. The last three sections are conclusions, acknowledgments and references.

\section{Application of Neuro-Fuzzy Systems to Technical Diagnostics and Measurement}

This section gives a survey on Neuro-Fuzzy system applications in the field of technical diagnostics and measurement. Different Neuro-Fuzzy architectures are named here, their history and a more detailed description are presented in the next sections. At the end of this section a table is also presented which gives a comprehensive overview of these applications and their fields categorically.

\section{1. $90 \mathrm{~s}$}

In the early 90s Neuro-Fuzzy was still a new concept to be shaped by different implementations and applications. In these years a relatively small amount of Neuro-Fuzzy application was published and naturally these were unique approaches rather than utilisation of existing solutions. For example among the pioneers, Ayoubi presented a structure that models the fuzzy inference mechanism based on neural units [1]. He tested the system on two real-world problems: monitoring the state of a turbocharger and supervision of air pressure in vehicle wheels. The implemented model proved to be efficient when the problem space is lowdimensional; however, when it had significantly more dimensions, Multi-Layer Perceptron (MLP) performed far better than the fuzzy inference mechanism.

Zhang and Morris also used a Neuro-Fuzzy solution for fault diagnosis of continuous stirred tank reactor process [2]. The chosen test problem is well known 
about its highly nonlinear dynamics which is a result of the phenomenon that process gain changes drastically with any operating condition modification. The network applied for this problem consists of 4 layers: an input layer, a fuzzification layer, a hidden layer and an output layer. The input layer has 14 neurons, because the system has 14 measured signal values, the fuzzification layer has 3 neurons for each input neuron, because each input information is ordered to 3 individual fuzzy membership function, the hidden layer has 10 neurons representing 10 fuzzy rules and the output layer has 11 neurons, each corresponding to a particular fault. They achieved much better performance than with a conventional feed forward neural network while the system also provided a more interpretable structure.

\section{2. $2000 \mathrm{~s}$}

Neuro-Fuzzy systems became more widespread in the 2000s especially in technical diagnostics and measurement. For example Sakuntala Mahapatra et al. built such systems for adaptive filtering of oscillatory signals [3]. The used model proved to be more efficient than other alternative fuzzy adaptive systems; moreover, it can be used for on-line monitoring of signals, independently whether they are described by linguistic variables or crisp variables.

Frey et al. used a Neuro-Fuzzy model to control a rotary hammer drill [4]. For solving this problem the authors had to find the optimal settings of rotational speed and strike rate to achieve optimal drill penetration. A self-learning Neuro-Fuzzy model was developed to intelligently control these two variables during the drilling process to achieve optimal performance.

Detecting the onset of damage in gear systems was the goal of Wang et al., for which they developed a Neuro-Fuzzy based diagnostic system [5]. The diagnosis of the gear system is conducted gear-bygear, which means that for every gear there is a separated Neuro-Fuzzy model. Each model has three inputs and one output: the inputs are reference functions that reduce the feature dimensions, i.e. they aggregate multiple features of the real system to one variable; the output is the condition of the gear, which can be normal or damaged. To train the implemented model they proposed a constrainedgradient-reliability algorithm which can effectively update the membership function parameters and set the rule weights.

Evsukoff and Gentil created a recurrent NeuroFuzzy system for fault detection and isolation in nuclear reactors [6]. In their model a fuzzification module is linked to a neural network based inference module which was adapted to recognize related faults based on the process variables.
One of the first and probably most widespread Neuro-Fuzzy architecture is the Adaptive-Networkbased Fuzzy Inference System (ANFIS) which has similar accuracy as the Multi-Layer Perceptron (MLP) which makes it ideal for function approximation. This architecture was used for mechanical fault diagnostics of induction motors with variable speed drives by Sadeghian and $\mathrm{Wu}$ [7]. The authors managed to significantly reduce the system complexity and learning duration of the network by using multiple ANFIS units in their model.

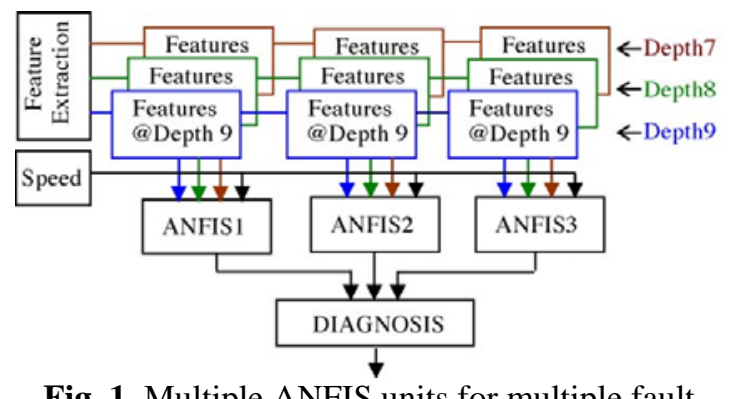

Fig. 1. Multiple ANFIS units for multiple fault diagnostics [7]

Fig. 1. shows the multiple ANFIS units where each one is responsible for detecting a specific fault type as these fault types have different feature coefficients. This modular structure provides an easy way to make extensions for detecting other fault types and also has the advantage that the units can be easily trained due to their simplicity. In another application Lei et al. used multiple ANFIS combination with genetic algorithm for fault diagnostics of rotating machinery [8]. They implemented a classifier system where the features describing the problem were divided into six predetermined and separated groups and individual Neuro-Fuzzy classifiers were constructed for each group. The final classification result of the system is the weighted average of the individual groups. During training, genetic algorithm was applied for optimising these weights. This method can yield better classification result than the member classifiers individually.

Amaral et al. applied a diagnostic technique based on the identification of a specified current pattern for detection of motor stator fault and used a NeuroFuzzy model for an image feature extraction based identification [9]. They used the Neuro-Fuzzy strategy to get a better linguistic knowledge about the underlying fault detection and diagnosis process.

Machinery malfunctions often reduce productivity and increase maintenance costs in various industrial fields. Zio and Gole proposed a Neuro-Fuzzy approach to solve fault diagnosis of rotating machinery by pattern classification while obtaining a model which remained easily interpretable [10]. 


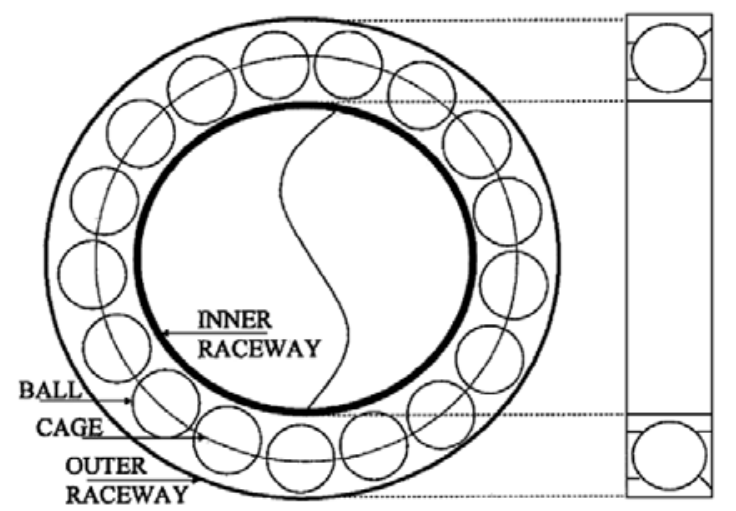

Fig. 2. A sketch of a bearing assembly [10]

Fig. 2. shows components of a bearing assembly which can be damaged leading to malfunction. For the diagnosis of these components the authors created a Neuro-Fuzzy algorithm which consists of multiple modules. First, an initial set of fuzzy rules are determined, where the initial large number of rules is reduced with a heuristic solution based on the firing strength of each rule. Then the forward algorithm calculates the relative strengths of the rules and the next module uses these values for creating new rules if necessary. The optimisation module tunes the parameters of the member functions and finally, a pruning is applied to reduce the size of the rule set. After the initial set of rules has been established, the algorithm repeats itself iteratively until the desired accuracy is reached.

Chen, Roberts and Weston used Neuro-Fuzzy Systems for fault detection and diagnostics of railway track circuits [11].

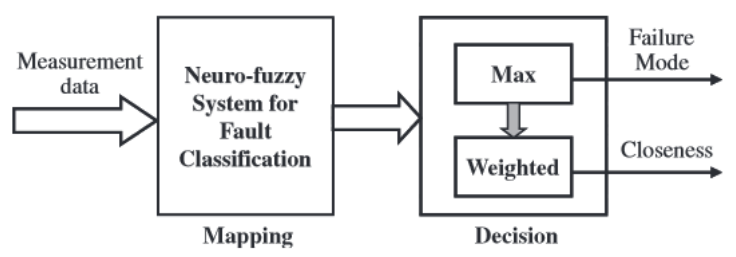

Fig. 3. Scheme of neuro-fuzzy for fault detection and diagnosis [11]

Fig. 3. Shows the scheme of the implemented model where the measurement data associated with different operating conditions is mapped to each failure mode. In their solution, they use a generalized version of the ANFIS to support multiple outputs. Each one of the eleven outputs corresponds to a condition (1 healthy and 10 faulty condition) while eight current and voltage measurements are used as the input variables.

Another research was aiming at Neuro-Fuzzy based state modelling of a flexible robotic arm using real sensor data [12]. The authors also utilized improved
Particle Swarm Optimization (PSO) to improve the model's learning capability. Via this solution, they showed that PSO can be effectively employed for developing industrial model-based control schemes.

\subsection{Nowadays}

Nowadays the emphasis of the research of NeuroFuzzy systems is concentrating mainly on their applications on wide practical fields. For instance Guzinski et al. presented a diagnostic system for shaft misalignment detection based on the ANFIS model [13]. They used this system in an adjustable speed sensorless induction motor drive where the model is based on the analysis of the stator current, motor speed and load torque processing. The results showed that it can effectively indicate the shaft misalignment. Karimi and Salahshoor also used the ANFIS model and combined it with Principal Component Analysis (PCA) for fault detection and diagnosis of distillation column [14]. They used PCA to extract the most informative features and at the same time reduce dimensionality of the measured data then fed the reduced data to ANFIS to discriminate the occurred fault. They demonstrated the effectiveness of the proposed method via extensive conducted tests in a distillation column benchmark.

Wali et al. compared intelligent controllers such as Fuzzy and Neuro-Fuzzy in the task of monitoring and control of novel advanced microwave biodiesel reactors [15].

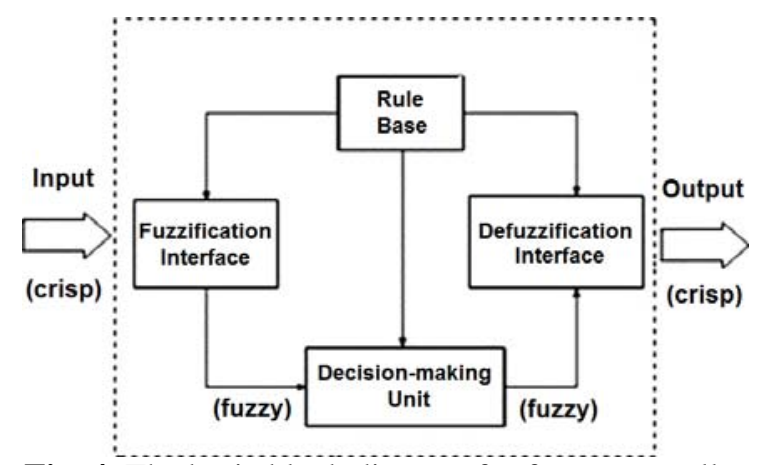

Fig. 4. The basic block diagram for fuzzy controller [15]

Fig. 4. shows the block diagram of the fuzzy controller which was applied for temperature control inside the reactor. The authors found that the ANFIS controller is more robust to parameter variations while a pure fuzzy solution is able to regulate in minimal overshoot. ANFIS model was used by Eristi for fault diagnosis of series compensated transmission lines, too [16]. 


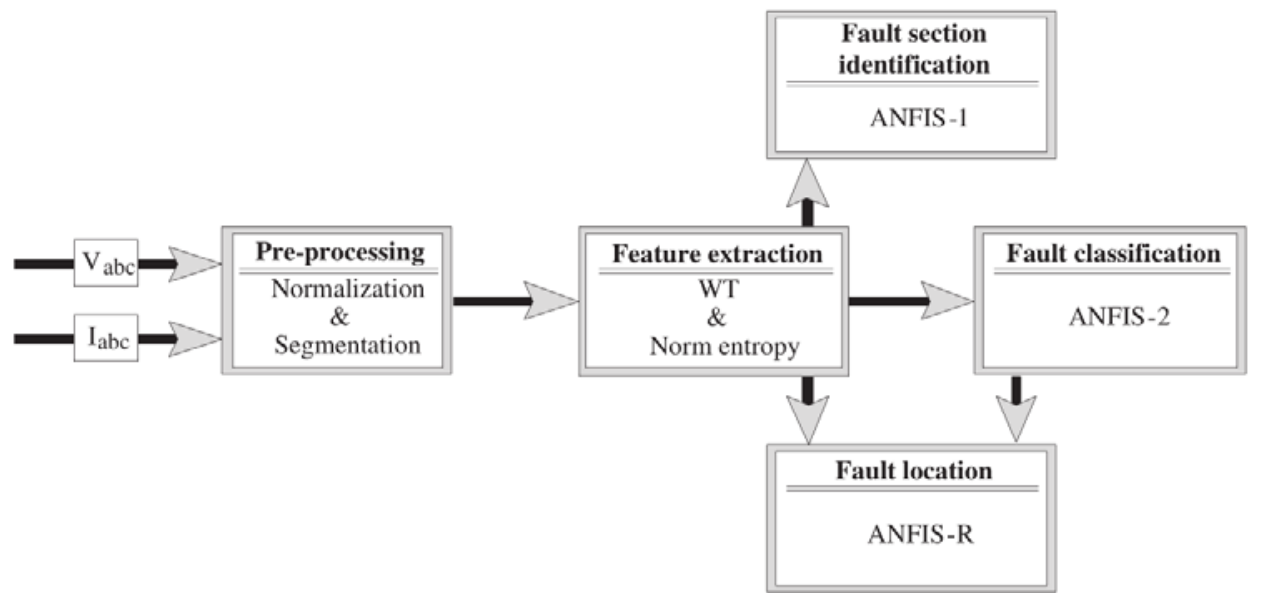

Fig. 5. Main structure of the proposed algorithm for fault diagnosis, classification and location isolation [16]

Fig. 5. shows the structure of the algorithm where $\mathrm{V}_{\mathrm{abc}}$ denotes the Voltage signal and $\mathrm{I}_{\mathrm{abc}}$ denotes the Current signal. Eristi utilized Wavelet Transformation (WT) and Norm entropy to achieve effective feature extraction of the fault signals. To test the proposed method an extensive data set of 23436 fault cases were used and the results showed that the algorithm is effective and robust to parameter variations.

Concentration estimation of volatile organic compounds was the goal of Jha et al. [17]. They built multiple models to measure and compare their performance on the problem. They found that the Neuro-Fuzzy system (ANFIS) outperformed both the clustering based fuzzy inference system and the ANN method and concluded that it is due to the fact that ANFIS inherits the advantages of both of the other models.

One of the major factors in catalytic performance is the size of the catalyst. In the next application the authors used response surface methodology and the ANFIS model to quantify the effects of physical characteristics of magnetite on Fenton-like oxidation efficiency of methylene blue [18]. For petroleum products monitoring Roshani et al. applied the ANFIS model to predict fluid density for a gamma ray densitometer [19].

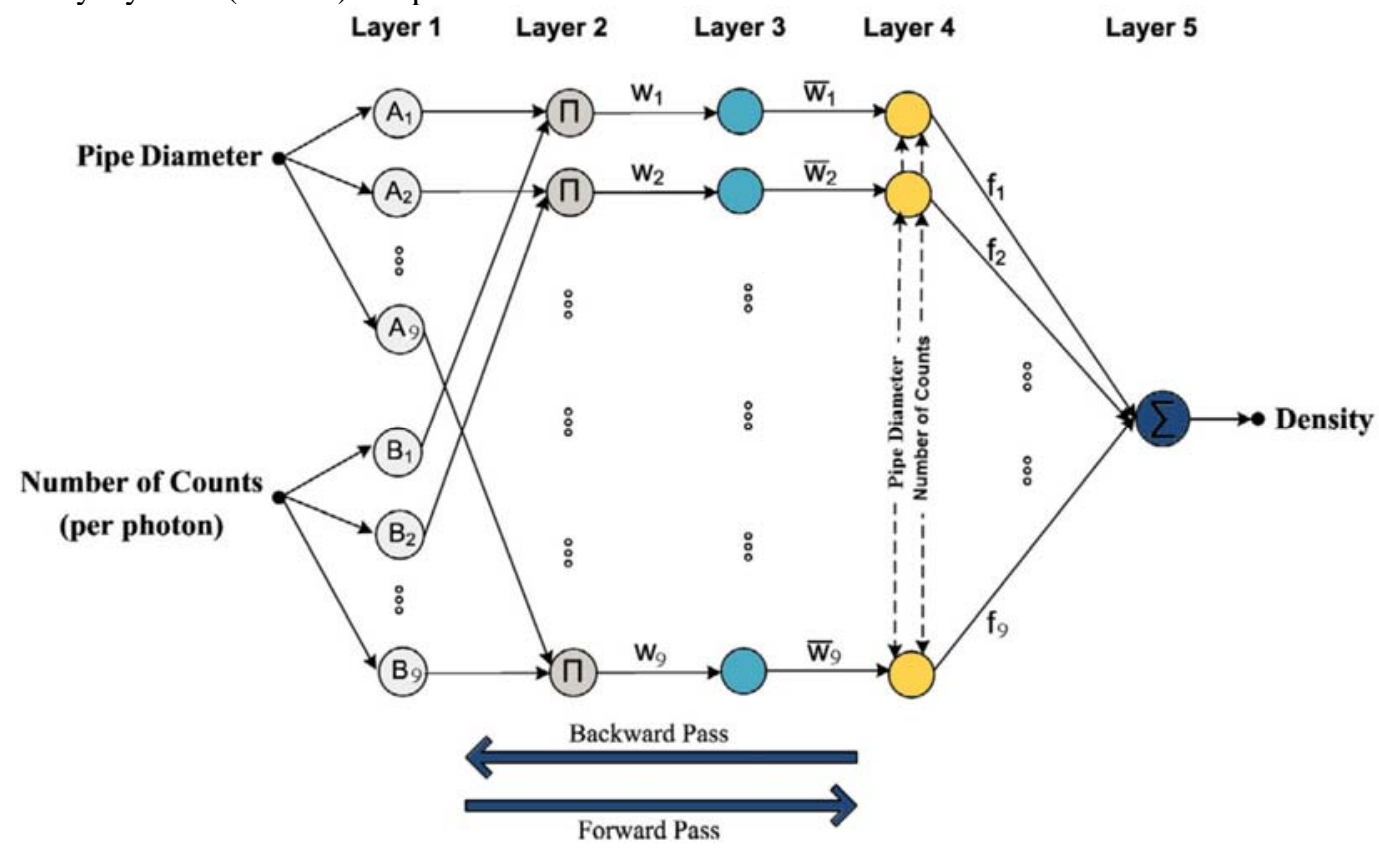

Fig. 6. The proposed ANFIS structure to predict fluid density for a gamma ray densitometer [19]

Fig. 6. shows the proposed model where $A_{1}-A_{9}$ denotes the membership functions of the pipe diameter variable and $\mathrm{B}_{1}-\mathrm{B}_{9}$ denotes the membership functions of the number of counts (per photon) variable. $\mathrm{W}_{1}-\mathrm{W}_{9}, \mathrm{~W}_{1}-\mathrm{W}_{9}$ and $\mathrm{f}_{1}-\mathrm{f}_{9}$ denotes the output values of the corresponding nodes. The authors simulated the operation of the densitometer device to obtain the testing dataset and after application of the model they concluded that it can estimate the fluid density with high accuracy. 
In many cases very different application fields are targeted by Neuro-Fuzzy solutions as in the case of another ANFIS model which was used to detect alterations in sleep EEG activity during hypopnoea episodes by Übeyli et al. [20]. The authors used the ANFIS for classification and they performed feature extraction by computing of wavelet coefficients. In their case four models was used: three were fed directly by measured data on the electrodes and the fourth had the purpose of improving diagnostic accuracy by gaining its inputs from the outputs of the other three systems. Lee and Lim also used hybrid fuzzy neural methods to compare Deep Brain Stimulation (DBS) and levodopa as two treatments of Parkinsonian resting tremors [21].

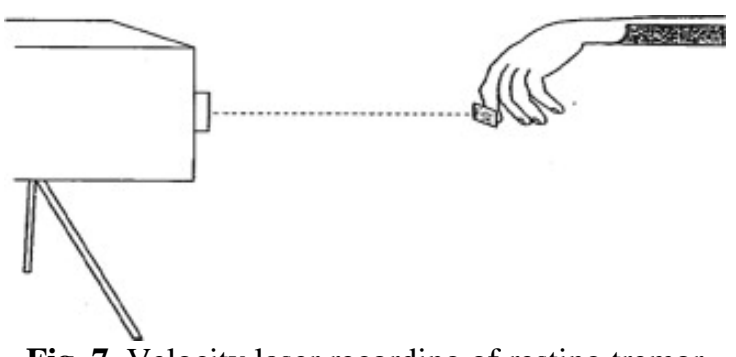

Fig. 7. Velocity laser recording of resting tremor [21]

Fig. 7. shows a method for recording resting tremors which was applied to 16 subjects to build a dataset. The study not only showed that DBS is more effective than levodopa but it did it in a way that is less time-consuming and less expensive than MRI and other medical expert dependant solutions. In another case ANN and Neuro-Fuzzy based models were applied to classify earthquake damages in buildings which can be utilized to help engineers decide whether some structures are remained safe or not [22]. The presented classification models used 20 seismic parameters of accelerograms to estimate 4 damage categories.

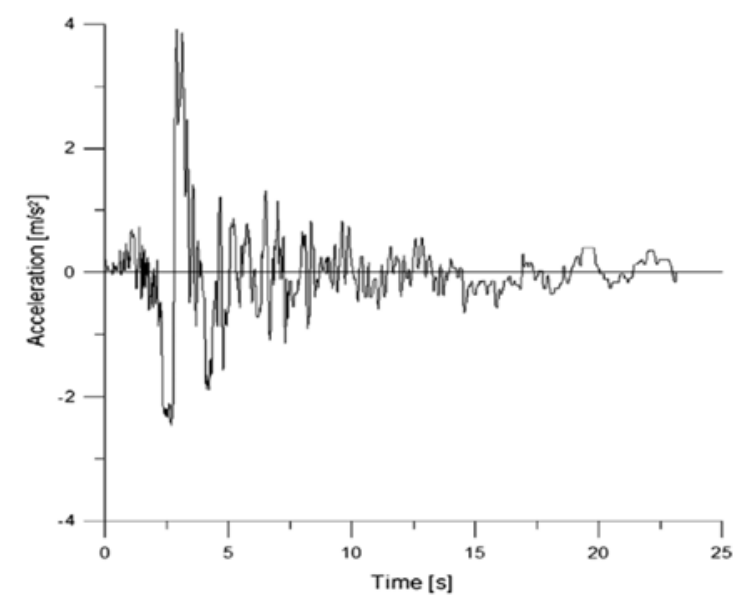

Fig. 8. Artificial seismic accelerogram processed to classify earthquake damages in buildings [22]
Fig. 8. shows a typical artificial accelerogram from which the 20 parameters were extracted. The proposed models were able to produce 98\% recognition rate and thus their application can be tested in real circumstances.

Benyamin Khoshnevisan et al. adopted an ANFIS and an ANN based system to predict potato yield from energy inputs [23]. The purpose of the study was to create model which helps farmers to estimate the level of production in advance and make an appropriate plan for the future. The authors evaluated various topologies to find the optimal one; moreover they compared the ANN and ANFIS models and found that the latter one is more capable of overcoming the problem of inconsistent data because of its rule based architecture.

Another application aimed at prediction of vertical stress transmission in real soil profile using ANFIS [24]. The authors built multiple models with different membership function types and for this specific dataset the gauss membership function proved to be the most efficient. Moharana et al. also used the ANFIS model to estimate the roughness coefficients of a meandering channel [25]. This is a complex problem because the coefficients depend on many hydraulic, geometric and roughness parameters. They compared the result with earlier studies and concluded that the Neuro-Fuzzy model is more effective than others in terms of estimation accuracy.

There is a wide variety of other applications where this kind of systems was successfully implemented from the fields of biology and environment to fault detection and diagnostics as by Kar et al. [26].

Neuro-Fuzzy applications are widely used for technical diagnosis and measurement purposes; however, neural and fuzzy methods are often used individually, too. For instance Bilski used an artificial intelligence-based model for diagnostics of analog systems [27]. He preprocessed the training and testing data sets using statistical methods to minimize the amount of information to be measured in order to optimize the performance of the Artificial Neural Network (ANN) diagnostic modules.

In another application Catelani and Ciani analysed the problem of disturbance induced by high energy particles on electronic devices and developed a model to determine whether a system respond to specific requirements [28].

\subsection{Overview of Neuro-Fuzzy System applications to Technical Diagnostics and Measurement}

Table 1 gives a comprehensive structure to all overviewed applications appointing the main functionalities and application fields and the related publications. 
Table 1

Applications functionalities, with their fields and related publications

\begin{tabular}{|c|c|c|}
\hline Functionality & Application field & Authors and Reference \\
\hline \multirow{4}{*}{$\begin{array}{l}\text { Monitoring and } \\
\text { supervision }\end{array}$} & Turbocharger state, air pressure of vehicle wheels & Ayoubi [1] \\
\hline & Fenton-like oxidation efficiency & Pouran et al. [18] \\
\hline & fluid density for a gamma ray densitometer & Roshani et al. [19] \\
\hline & Analogue systems & Bilski [27] \\
\hline \multirow{9}{*}{ Fault diagnosis } & Continuous stirred tank reactor process & Zhang and Morris [2] \\
\hline & Gear systems & Wang et al. [5] \\
\hline & Nuclear reactors & Evsukoff and Gentil [6] \\
\hline & Induction motors & $\begin{array}{r}\text { Wu [7] } \\
\text { Guzinski et al. [13] }\end{array}$ \\
\hline & Rotating machinery & $\begin{array}{r}\text { Lei et al. [8] } \\
\text { Zio and Gole [10] }\end{array}$ \\
\hline & Railway track circuits & Chen et al. [11] \\
\hline & Distillation column & Karimi and Salahshoor [14] \\
\hline & Series compensated transmission lines & Eristi [16] \\
\hline & Disturbance induced by high energy particles & Catelani and Ciani [28] \\
\hline Adaptive filtering & Oscillatory signal & Sakuntala Mahapatra et al. [3] \\
\hline \multirow{2}{*}{ Control } & Rotary hammer drill & Frey et al. [4] \\
\hline & Microwave biodiesel reactors & Wali et al. [15] \\
\hline Pattern identification & Motor stator & Amaral et al. [9] \\
\hline System state modelling & Flexible robotic arm & Amitava et al. [12] \\
\hline \multirow{4}{*}{ Variable estimation } & Volatile organic compounds & Jha et al. [17] \\
\hline & Potato yield & Khoshnevisan et al. [23] \\
\hline & Soil profile & Hamid Taghavifar et al. [24] \\
\hline & Meandering channel & Moharana et al. [25] \\
\hline \multirow{2}{*}{$\begin{array}{l}\text { Variable change } \\
\text { detection }\end{array}$} & Sleep EEG activity during hypopnoea episodes & Übeyli et al. [20] \\
\hline & Parkinsonian resting tremors & Lee and Lim [21] \\
\hline Damage classification & Earthquake damages in buildings & Alvanitopoulos et al. [22] \\
\hline
\end{tabular}

\section{Prelude of Neuro-Fuzzy Systems}

This section discusses the techniques that provided the theoretical basis and allowed the concept of Neuro-Fuzzy system to be formed. These techniques are the Neural Networks and the Fuzzy Systems which will be presented in the following paragraphs.

\subsection{Artificial Neural Networks (ANNs)}

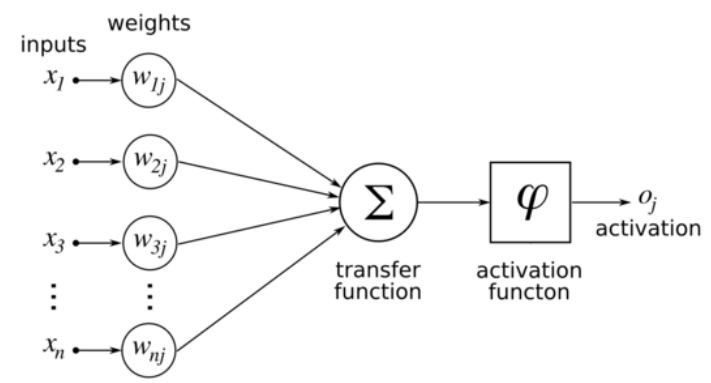

Fig. 9. An artificial neuron

The concept of the ANN was established seven decades ago and, as the name suggests, it was inspired by the behaviour of biological neural networks inside the human brain. An ANN implements the functionality of the biological neural networks by building up a network of autonomous computational units (neurons) and connecting them via weighted links defined by the first pioneers W. S. McCulloch and W. Pitts [29]. Such a computational unit e.g. an artificial neuron is shown on Fig. 9. It consists of input links (special neurons sometimes don't have any input links), a transfer function, an activation function and an optional memory component while the weighted links between them are represented by real numbers. In Fig. 9. $x_{1}-x_{n}$ denotes the input values of the neuron, $o_{j}$ denotes the output value and $w_{1 j}-w_{n j}$ denotes the weights of the input links. The transfer function aggregate the outputs of the other neurons connected via the input links weighted with the strength of the links. The activation function produces the output value based on the output of the transfer function and the memory serves as a container to store previous states of the neuron (in many cases it is not used or only a part of the state is stored). When a neuron fires the signal propagates through the output links to the connected neurons and the weight of a link 
(represented by a real number) determines the strength of the connection and weakens the signal accordingly.

In 1957 Frank Rosenblatt created the perceptron algorithm for supervised classification of an input into one of two possible outputs [30]. This is a type of linear classifier and at the time, only the singlelayer perceptron could be trained.

18 years later Werbos created the backpropagation algorithm for training the MLP [31].

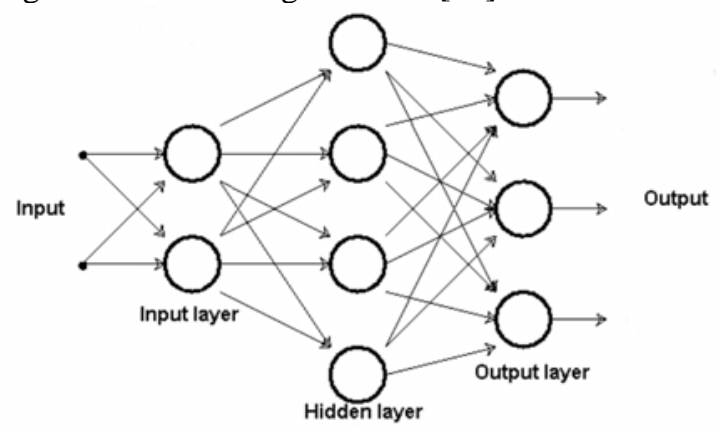

Fig. 10. The MuliLayer Perceptron model

Fig. 10. shows an MLP model where the neurons are organized into layers and each layer is fully connected with the next one. Supervised training of an MLP means repeated adjustment of the weight of each link to receive more and more favourable output on specific neurons (output neurons) while stimulating other neurons (input neurons). The backpropagation algorithm achieves this by calculating the derivatives of the network's error with respect to all of its weights and adjusting the weights to a position where, based on the derivatives, the error is smaller e.g. moving the weights in the direction of the descent of the derivatives where the error is a measure of the difference between the network's output and the target values for the same input.

After 1975 the MLP became more popular and widespread and during the years many other, but not so highly popular ANN model types were defined, moreover, currently the basic research in the field of neural networks is emphasising on the study of biological neural systems and define new learning algorithms and architectures that are maps of the biological brain systems. One of these new types is the Self-Organizing Map (SOM) which can be trained by unsupervised learning to produce a low dimensional representation of the input space [32]. They are mainly used for visualizing high dimensional data in low dimensional views.

Another type is the Recurrent Neural Network (RNN), they are different from the common feedforward networks as they allow circles in their structure e.g. some links propagate the signal back to such neurons that sent the original signal. This feature allows them to establish an internal memory which can be used to process arbitrary sequences of inputs.

Yet another class of ANNs is the Radial Basis Function Network (RBFN) which uses radial basis functions as the activation function of the neurons [33]. Their training algorithms are extended to adjust not only the network's weights but the activation function parameters, too. They have many uses such as function approximation, time series prediction, classification, etc.

Over the decades ANNs proved to be powerful computational models for solving complex estimation and classification problems as they are robust and are capable of high level generalization, moreover they can already handle incomplete data, too [34]. However no information can be extracted from a trained ANN about the connections between the parameters, e.g. a generic ANN model can only approximate the output parameters but cannot tell what kind of connections exist between the input and output parameters. This is a key disadvantage of the Neural Network model which led to the creation of Neuro-Fuzzy Systems.

\subsection{Fuzzy Systems}

Real life problems often have the tendency to be not discrete but continuous in nature. A somewhat special case of this phenomenon is to categorize objects or theoretical entities because in many cases, categories don't have precisely defined criteria of membership. To solve this problem Lofti Zadeh [35] introduced fuzzy set theory, where the membership of an element is no longer a binary state but a continuous value e.g. instead of saying that $a$ is an element of $A$ set and not element of $B$, we can say that a is an element of $A$ fuzzy set by 0.67 degree and element of $B$ by 0.23 degree.

Fuzzy logic is a type of logic that uses fuzzy sets to represent truth values and consequently it provides an effective way to represent human knowledge in a mathematical language. Fuzzy logic uses fuzzy inference rules which are able to process the continuous truth values and produce an also continuous output. Each rule has the form of

$$
\text { if }<\text { premise }>\text { then }<\text { consequent }>\text {, }
$$

that uses linguistics variables with symbolic terms. Each term represents a fuzzy set. The terms of the input space (typically 5-7 for each linguistic variable) compose the fuzzy partition. The fuzzy inference mechanism consists of three stages: in the first stage, the values of the numerical inputs are mapped by a function according to a degree of compatibility of the respective fuzzy sets; this operation can be called fuzzification. In the second stage, the fuzzy system processes the rules in accordance with the firing strengths of the inputs. In the third stage, the resultant fuzzy values are 
transformed again into numerical values; this operation can be called defuzzification. Essentially, this procedure makes possible the use fuzzy categories in representation of words and abstracts ideas of the human beings in the description of the decision taking procedure [36].

Fuzzy inference systems have two main type based on the mathematical calculation of the inference. These are the Mamdani type inference [37] and the Takagi-Sugeno-Kang (TSK) type inference [38]. A Mamdani type fuzzy rule can be described as

\section{if $\boldsymbol{A}$ is $\boldsymbol{X}_{\mathbf{1}}$ and $\boldsymbol{B}$ is $\boldsymbol{X}_{\mathbf{2}}$ then $\boldsymbol{C}$ is $\boldsymbol{X}_{3}$,}

where $\mathrm{A}, \mathrm{B}, \mathrm{C}$ are variables and $\mathrm{X}_{1}, \mathrm{X}_{2}, \mathrm{X}_{3}$ are fuzzy sets. In contrast to the Mamdani type, a TSK rule has the form of

$$
\text { if } \boldsymbol{A} \text { is } \boldsymbol{X}_{1} \text { and } \boldsymbol{B} \text { is } \boldsymbol{X}_{2} \text { then } \boldsymbol{C}=\boldsymbol{a} \boldsymbol{A}+\boldsymbol{b} \boldsymbol{B}+\boldsymbol{c} \text {, }
$$

where a, b and c are constants. As a result of the form of the rules the Mamdani type inference systems are more interpretable because both the premises and consequents of the rules are fuzzy sets while the Takagi-Sugeno-Kang types are more accurate and computationally efficient, e.g. they build up more accurate models, however, here, only the premises of the rules are fuzzy sets.

All in all, Fuzzy Systems have the advantage that the fuzzy rules, which store the information, are easily interpretable. Furthermore they provide a simple interface for extending the system with new information (by adding new rules) or manipulating the existing rules. The problem with Fuzzy Systems lies in the fact that they completely depend on the experts who design them. It only uses the information which were encoded in the system and cannot learn on its own and it is incapable of generalization. The described nature of Fuzzy Systems indicates that a fusion with ANNs may possibly lead to a new powerful computational model.

\section{Neuro-Fuzzy System Architectures}

The previous section briefly described the concept of the two main components building up a NeuroFuzzy system individually, so in this section the different architectures can be discussed to show how different approaches managed to combine ANNs with Fuzzy Systems. At the end of this section a table is also presented summarizing the advantages and drawbacks of each presented architecture.

\subsection{ANFIS Architecture}

One of the first Neuro-Fuzzy Systems was introduced by Jang in year 1993 [39][40]. This architecture is called ANFIS and it uses the TakagiSugeno-Kang inference system.

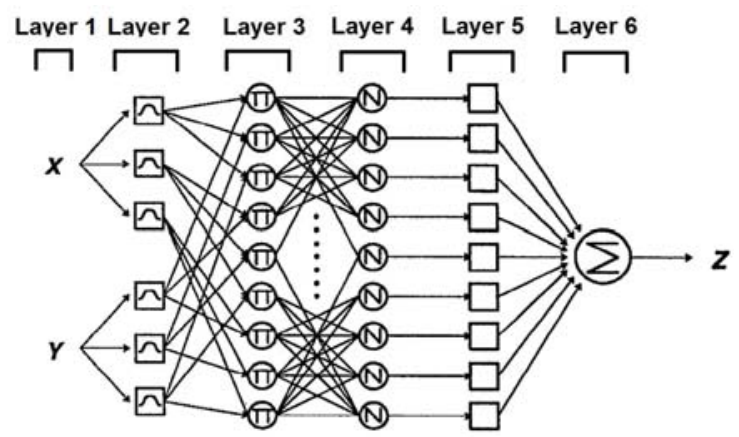

Fig. 11. The ANFIS architecture [39] where $x$ and $y$ denote the input variables and $\mathrm{z}$ denotes the output variable

Fig. 11. shows the ANFIS architecture consisting of six layers. The first layer contains two nodes for input $\mathrm{x}$ and $\mathrm{y}$, the second layer is responsible for mapping input values to the membership functions. The nodes of the third layer correspond to the fuzzy rules in the form of production functions; their output values are the firing strengths of each rule while the nodes in the fourth layer calculate the ratio to the sum of all rules' firing strengths. Defuzzification happens in the fifth layer and the sixth layer's output nodes sum their input values. Iterative learning of ANFIS is composed of two stages. In the first stage the parameters of the consequent functions (in the fifth layer) are tuned via a least mean square method. During the second stage the parameters of the premise functions (in the second layer) are adjusted by a backpropagation algorithm. These two stages are repeated iteratively for training of the system. It is also worth to mention that this model has the best estimation accuracy based on various benchmarking and application results.

\subsection{FALCON Architecture}

Approximately in the same time as ANFIS, the Fuzzy Adaptive Learning Control Network (FALCON) architecture was introduced, which is a system with five layers and uses Mamdani type inference [41].

Fig. 12. shows the FALCON architecture. Input nodes are located in the first layer; second layer has term nodes which represent the membership functions for the input values. Each node of the third layer acts as a fuzzy rule. The fourth layer also consists of term nodes; these represent the membership functions for the outputs. Finally the fifth layer is the output layer; here for every output there are two nodes: one is for training data which is the desired output and the other is for decision signal which is the actual output. 


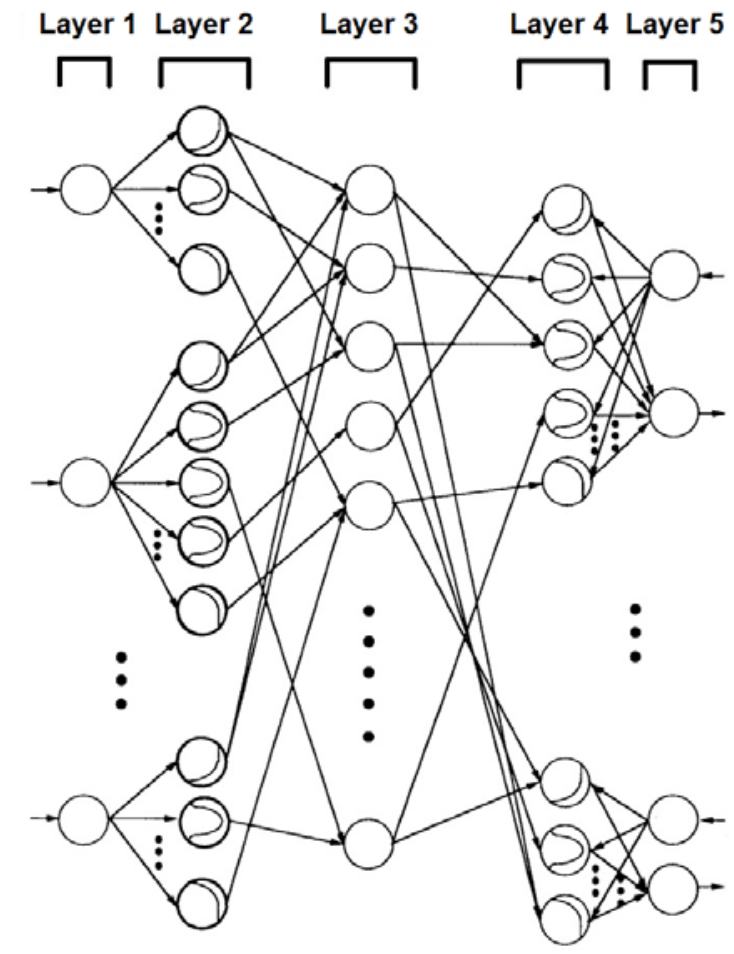

Fig. 12. The FALCON architecture

Training is done by a two-phase-algorithm. The first phase is responsible for finding the initial membership functions by a self-organized learning scheme. In the second phase the parameters of the membership functions are adjusted using supervised learning. During the training nodes and links can be deleted or merged reforming the structure of the network.

\subsection{GARIC Architecture}

Another early Neuro-Fuzzy model is the Generalized Approximate Reasoning based Intelligence Control (GARIC) system, which is composed of three components: the Action Selection Network (ASN), the Action Evaluation Network (AEN) and the Stochastic Action Modifier (SAM) [42].

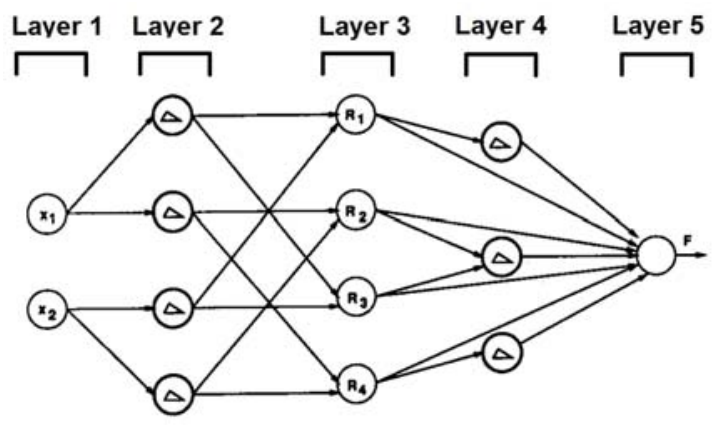

Fig. 13. The ASN component of the GARIC architecture [42]
Fig. 13. shows the ASN component. The ASN is a five layer network which is responsible for selecting an action based on the current state of the system using fuzzy inference. Input nodes are in the first layer and the second one holds the membership functions. Each node in the third layer represents a fuzzy rule and nodes of the forth layer correspond to consequent labels, e.g. if a consequent label is in a rule then there is a link between the label's node and the rule's node. The fifth layer's nodes calculate the real output values based on the rules' firing strength and the forth layer's outputs. The AEN component of this architecture is a simple feedforward network which predicts reinforcements based on the state variables of the system. And the SAM component stochastically generates an action from based on the recommendation of the AEN GARIC uses gradient descending and reinforcement learning to adjust its internal parameters.

\subsection{NEFCON Architecture}

In parallel with the models mentioned earlier, the Neural Fuzzy Controller (NEFCON) architecture had been created, which has three layers and implements a Mamdani type inference system [43].

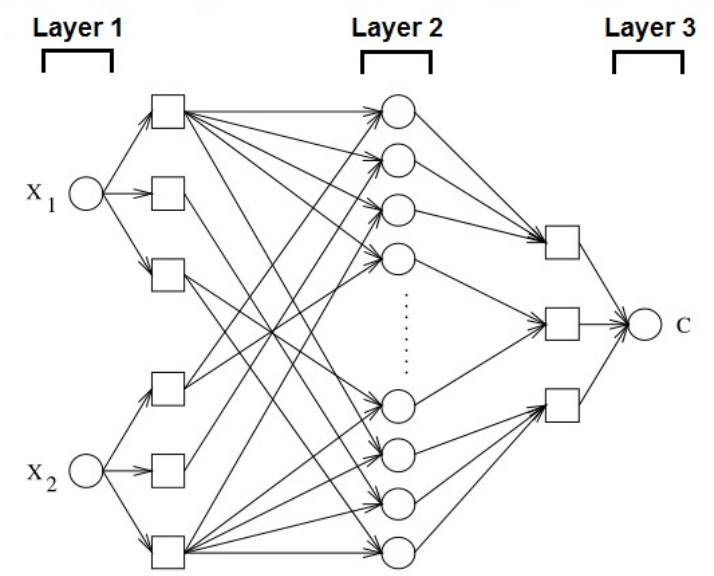

Fig. 14. The NEFCON architecture where $x_{1}$ and $x_{2}$ denote the input variables and c denotes the output variable

Fig. 14. Shows the NEFCON architecture where the circles indicate the nodes which are forming the layers and the rectangles indicate the shared weights of the network. The first layer consists of the input nodes, in the second layer the nodes represent the fuzzy rules and the third layer holds the output nodes. In this architecture the links connecting the nodes are weighted with fuzzy sets. The learning procedure uses reinforcement learning with backpropagation algorithm to either learn the rule base from the beginning or to optimise an initially defined rule base. Two other systems were developed based on NEFCON which are 
specialized versions of the original architecture. These systems are the NEFCLASS [44] which is specialized in classification problems and the NEFPROX [45] which was created for function approximation.

\subsection{SONFIN Architecture}

Self-Constructing Neural Fuzzy Inference Network (SONFIN) is a Takagi-Sugeno-Kang-type fuzzy rule-based model which consists of six layers [46].

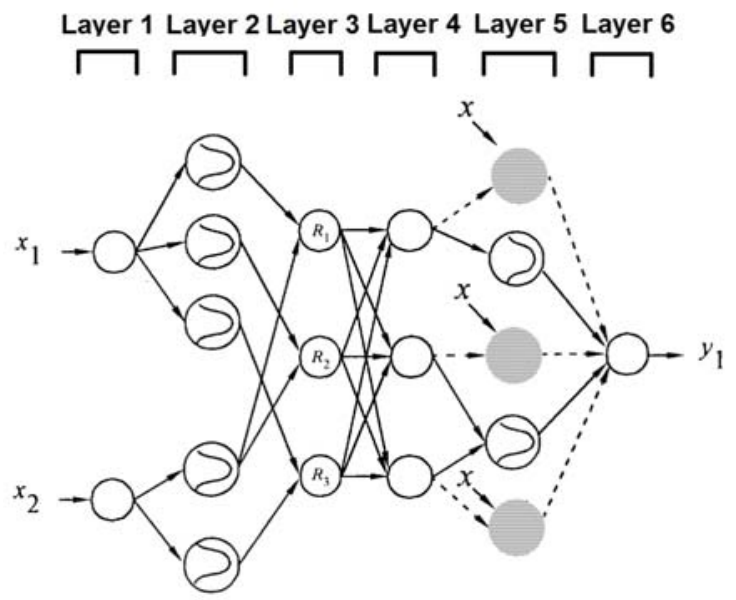

Fig. 15. The SONFIN architecture [46] where $x_{1}$ and $\mathrm{x}_{2}$ denote the input variables, $\mathrm{y}_{1}$ denotes the output variable, $R_{1}-R_{3}$ denotes the rule nodes and $x$ represents the input vector

Fig. 15. shows the SONFIN architecture which, in fact, is similar to the ANFIS. Layer 1-4 and 6 are functioning as they are in the ANFIS architecture. The fifth, consequent layer can hold two types of nodes. The first type represents the fuzzy sets by membership functions while the second type is optional and gains its inputs from the first and fourth layer.

Constructing of SONFIN happens concurrently by a structure and a parameter learning method. The structure learning identifies both the precondition and consequent parts of the rules by minimizing the number of rules and membership functions for the input and by optimally generating new membership functions for the output variables. Parameter learning uses LMS or RLS algorithms to adjust consequent parameters and backpropagation for precondition parameters.

\section{6. dmEfuNN Architecture}

The Dynamic Evolving Fuzzy Neural Network (dmEfuNN) is a system with five layers which uses the Takagi-Sugeno fuzzy inference mechanism [47]. The predecessors of this model are FuNN [48] and EFuNN [49] which both uses the Mamdani type inference.

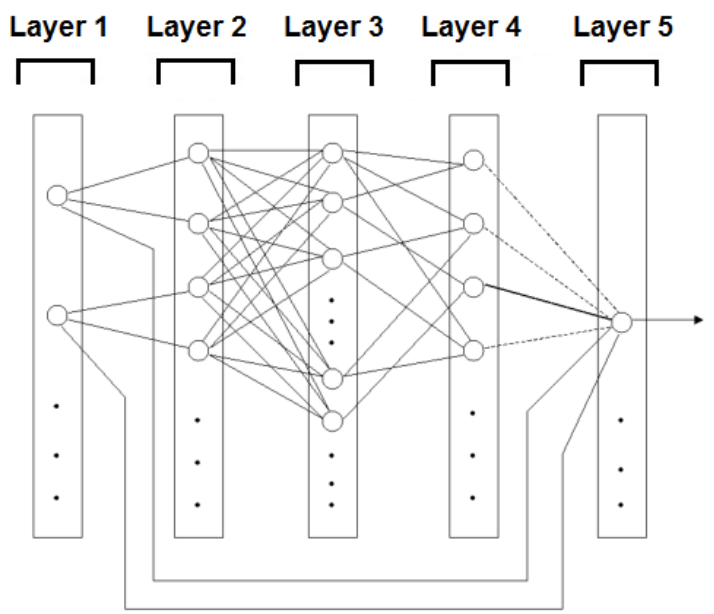

Fig. 16. The dmEfuNN architecture

Fig. 16. shows the dmEfuNN architecture. The first layer contains the input nodes and membership functions are in the second layer. Fuzzy rules are represented by the nodes in the third layer. Fourth layer selects a number of rules from the third layer which are the closest to the fuzzy inputs and the fifth layer does the defuzzification and produces real outputs.

The dmEfuNN can optimize global generalization error and local generalization error in contrast to MLP and ANFIS which can only optimize global error. As the name suggests the number of nodes and links in the structure can dynamically increase or decrease during the on-line learning while offline training uses a given structure and optimizes internal parameters. 


\subsection{Comparison of the Neuro-Fuzzy architectures}

Table 2 gives a comprehensive comparison to all overviewed Neuro-Fuzzy system types appointing the main advantages and drawbacks of each architecture.

Table 2

Advantages and drawbacks of the presented architectures.

\begin{tabular}{|c|c|c|}
\hline Architecture & Advantages & Drawbacks \\
\hline ANFIS & $\begin{array}{l}\text { - As it implements a Takagi-Sugeno- } \\
\text { Kang inference mechanism, it is a } \\
\text { very accurate model (most accurate } \\
\text { among the presented architectures and } \\
\text { more accurate than Multi-Layer } \\
\text { Perceptron and pure Fuzzy systems) }\end{array}$ & $\begin{array}{l}\text { - } \quad \text { Cannot handle multiple output systems } \\
\text { - Only fully defined structures can be trained } \\
\text { - } \quad \text { There is no dynamic rule creation or reduction }\end{array}$ \\
\hline FALCON & $\begin{array}{l}\text { - It has a learning phase for building up } \\
\text { its initial structure (number of rules } \\
\text { and membership functions can be } \\
\text { determined via training) } \\
\text { - Its Mamdani type inference makes it } \\
\text { more interpretable }\end{array}$ & $\begin{array}{l}\text { - Its Mamdani type inference makes it less } \\
\text { accurate especially for parameter estimation }\end{array}$ \\
\hline GARIC & $\begin{array}{l}\text { - It is one of the earliest presented } \\
\text { Neuro-Fuzzy system }\end{array}$ & $\begin{array}{l}\text { - Isn't a single model but consists of multiple } \\
\text { components }\end{array}$ \\
\hline NEFCON & $\begin{array}{l}\text { - It is able to reduce the number of } \\
\text { rules during its training } \\
\text { - Its Mamdani type inference makes it } \\
\text { more interpretable }\end{array}$ & $\begin{array}{l}\text { - Its Mamdani type inference makes it less } \\
\text { accurate especially for parameter estimation }\end{array}$ \\
\hline SONFIN & $\begin{array}{l}\text { - There are no initial rules but rules are } \\
\text { created and adapted as on-line } \\
\text { learning proceeds via simultaneous } \\
\text { structure and parameter identification } \\
\text { - The number of generated rules and } \\
\text { membership functions is small even } \\
\text { for modelling of a sophisticated } \\
\text { system }\end{array}$ & $\begin{array}{l}\text { - Not as accurate as the ANFIS (which has the } \\
\text { most similar structure to this) }\end{array}$ \\
\hline dmEfuNN & $\begin{array}{l}\text { - During its on-line training it can } \\
\text { increase or decrease the number of } \\
\text { rules in the system }\end{array}$ & - Not as accurate as the ANFIS \\
\hline
\end{tabular}

\section{Conclusions}

Different applications of Neuro-Fuzzy Systems were discussed to show their high potential in technical diagnostics and measurement. This survey summarizes in a comprehensive overview the Neuro-Fuzzy applications in technical diagnostics and measurement with appointing the generalized, main, typical functionalities and with highlighting their great variety of application areas.

These systems are successful because of their nature that they reveal the nature of the important interdependence between the parameters of the modelled system while they are, in fact, powerful approximators. Their ability to discover connections between parameter intervals can be extremely useful when applying the model for diagnostic and control tasks because entirely different rules can be used to specific subsets of the problem, e.g. these systems have the capability to work differently in different parts of the parameter space providing a more detailed and somehow distributed model instead of a general solution.

The paper briefly reviewed the concept of Artificial Neural Networks and Fuzzy Systems as computational models and how they inspired the creation of Neuro-Fuzzy Systems. As it was discussed this fusion can unite the generalization capabilities of Neural Networks with the easy interpretability and high expressive power of fuzzy rules in an effective way.

Six different architectures were presented and it can be concluded that these are the most important ones although there are other structure variations, too. Usually each architecture organizes its nodes a slightly different way and consequently they use specific learning algorithms which are adapted to the different structures. The different Neuro-Fuzzy models were also compared and a table is also presented summarizing the advantages and 
drawbacks of each presented architecture.

All in all it can be said that ANFIS architecture is the most popular and widespread among the NeuroFuzzy systems for various applications in the field of diagnostics, control or for medical research, civil engineering, etc. This is mainly because the ANFIS model has higher accuracy than the other NeuroFuzzy model types which compensates its less interpretable structure.

\section{Acknowledgments}

The authors acknowledge the support of grants of the Fraunhofer Project Center for Production Management and Informatics at SZTAKI, Budapest, Hungary and the Highly industrialised region on the west part of Hungary with limited R\&D capacity: Research and development programs related to strengthening the strategic future-oriented industries manufacturing technologies and products of regional competences carried out in comprehensive collaboration, VKSZ_12-1-2013-0038, with iKOMP acronym.

\section{References}

[1] M. Ayoubi, "Fuzzy systems design based on a hybrid neural structure and application to the fault diagnosis of technical processes", Control Engineering Practice, vol. 4: (1), pp. 35-42, 1996.

[2] J. Zhang, J. Morris, "Process modelling and fault diagnosis using fuzzy neural networks", Fuzzy Sets and Systems, vol. 79, pp. 127-140, 1996.

[3] Sakuntala Mahapatra, Santanu K. Nayak, Samrat L. Sabat, "Neuro fuzzy model for adaptive filtering of oscillatory signals", Measurement, vol. 30, pp. 231-239, 2001.

[4] C. W. Frey, A. Jacubasch, H.-B. Kuntze, „Smart neuro-fuzzy based control of a rotary hammer drill“, IEEE International Conference on Robotics and Automation, vol. 2, pp. 26342639, 2003.

[5] W. Wang, F. Ismail, A. F. Golnaraghi, “A neuro-fuzzy approach to gear system monitoring”, IEEE Transactions on Fuzzy Systems, vol. 12: (5), pp. 710-723, 2004.

[6] A. Evsukoff, S. Gentil, "Recurrent neurofuzzy system for fault detection and isolation in nuclear reactors", Advanced Engineering Informatics, vol. 19, pp. 55-66, 2005.

[7] Z. Ye, A. Sadeghian, B. Wu, "Mechanical fault diagnostics for induction motor with variable speed drives using Adaptive Neurofuzzy Inference System”, Electric power System Research, vol. 76, pp. 742-752, 2006.

[8] Y. Lei, Z. He, Y. Zi, Q. Hu, "Fault diagnosis of rotating machinery based on multiple
ANFIS combination with GAs”, Mechanical Systems and Signal Processing, vol. 21: (5), pp. 2280-2294, 2007.

[9] T. G. Amaral, V. F. Pires, J. F. Martins, A. J. Pires, M. M. Crisóstomo, "Image processing to a neuro-fuzzy classifier for detection and diagnosis of induction motor status fault", $33^{\text {rd }}$ Annual Conference of the IEEE Industrial Electronics Society, Taipei, Taiwan, 2007.

[10] E. Zio, G. Gola, “A neuro-fuzzy technique for fault diagnosis and its application to rotating machinery", Reliability Engineering and System Safety, vol. 94, pp. 78-88, 2009.

[11] J. Chen, C. Roberts, P. Weston, "Fault detection and diagnosis for railway track circuits using neuro-fuzzy systems”, Control Engineering Practice, vol. 16: (5), pp. 585596, 2008.

[12] Amitava Chatterjee, Ranajit Chatterjee, Fumitoshi Matsuno, Takahiro Endo, "Neurofuzzy state modelling of flexible robotic arm employing dynamically varying cognitive and social component based PSO”, Measurement, vol. 40, pp. 628-643, 2007.

[13] J. Guzinski, H. Abu-Rub, A. Iqbal, S. M. Ahmed, "Shaft misalignment detection using ANFIS for speed sensorless AC drive with inverter output filter”, IEEE International Symposium on Industrial Electronics, Gdansk, Poland, 2011.

[14] I. Karimi, K. Salahshoor, “A new fault detection and diagnosis approach for a distillation column based on a combined PCA and ANFIS scheme”, $24^{\text {th }}$ Chinese Control and Decision Conference, Taiyuan, China, 2012.

[15] W. A. Wali, K. H. Hassan, J. D. Cullen, A. Shaw, A. I. Al-Shamma's, "Real time monitoring and intelligent control for novel advanced microwave biodiesel reactor", Measurement, vol. 46, pp. 823-839, 2013.

[16] Huseyin Eristi, "Fault diagnosis system for series compensated transmission line based on wavelet transform and adaptive neuro-fuzzy inference system”, Measurement, vol. 46, pp. 393-401, 2013.

[17] Sunil K. Jha, Kenshi Hayashi, R. D. S. Yadava, "Neural, fuzzy and neuro-fuzzy approach for concentration estimation of volatile organic compounds by surface acoustic wave sensor array”, Measurement, vol. 55, pp. 186-195, 2014.

[18] Shima Rahim Pouran, A. R. Abdul Aziz, Wan Mohd Ashri Wan Daud, Shahaboddin Shamshirband, "Estimation of the effect of catalyst physical characteristics on Fenton-like oxidation efficiency using adaptive neurofuzzy computing technique”, Measurement, vol. 59, pp. 314-328, 2015.

[19] G. H. Roshani, S. A. H. Feghhi, A. Adineh- 
Vand, M. Khorsandi, “Application of adaptive neuro-fuzzy inference system in prediction of fluid density for a gamma ray densitometer in petroleum products monitoring”, Measurement, vol. 46, pp. 3276-3281, 2013.

[20] E. D. Übeyli, D. Cvetkovic, G. Holland, I. Cosic, “Adaptive neuro-fuzzy inference system employing wavelet coefficients for detection of alterations in sleep EEG activity during hypopnoea episodes”, Digital Signal Processing, vol. 20, pp. 678-691, 2010.

[21] Sang-Hong Lee, Joon S. Lim, "Comparison of DBS and levopoda on resting tremor using a fuzzy neural network system”, Measurement, vol. 46, pp. 1995-2002, 2013.

[22] P. F. Alvanitopoulos, I. Andreadis, A. Elenas, "Neuro-fuzzy techniques for the classification of earthquake damages in buildings", Measurement, vol. 43, pp. 797-809, 2010.

[23] Benyamin Khoshnevisan, Shahin Rafiee, Mahmoud Omid, Hossein Mousazadeh, "Prediction of potato yield based on energy inputs using multi-layer adaptive neuro-fuzzy inference system”, Measurement, vol. 47, pp. 521-530, 2014

[24] Hamid Taghavifar, Aref Mardani, "Prognostication of vertical stress transmission in soil profile by adaptive neurofuzzy inference system based modelling approach”, Measurement, vol. 50, pp. 152159, 2014.

[25] S. Moharana, K. K. Khatua, "Prediction of roughness coefficient of a meandering open channel flow using Neuro-Fuzzy Inference System”, Measurement, vol. 51, pp. 112-123, 2014.

[26] S. Kar, S. Das, P. K. Ghosh, “Applications of neuro fuzzy systems: A brief review and future outline”, Applied Soft Computing, vol. 15, pp. 243-259, 2014.

[27] P. Bilski, "Data set processing for the optimization of the artificial intelligence-based diagnostic methods”, IMEKO TC10 Workshop on Technical Diagnostics, Florence, Italy, 2013.

[28] M. Catelani, L. Ciani, "Diagnostic and Error Correction System for Avionics Devices in Presence of Single Event Upset (SEU)”, IMEKO TC10 Workshop on Technical Diagnostics, Florence, Italy, 2013.

[29] W. S. McCulloch, W. Pitts, “A logical calculus of the ideas immanent in nervous activity”, Bulletin of Mathematical Biophysics, vol. 5, pp. 115-133, 1943.

[30] F. Rosenblatt, The Perceptron -- a perceiving and recognizing automaton, Cornell Aeronautical Laboratory, Report Number: 85460-1, 1957.

[31] P. J. Werbos, Beyond Regression: New Tools for Prediction and Analysis in the Behaviour Sciences, Ph. D Thesis, Harward University, Cambridge, 1974.

[32] T. Kohonen, Self-organized Formation of Topologically Correct Feature Maps, Biological Cybernetics, 43, pp. 59-69, 1982.

[33] D. S. Broomhead, D. lowe, Radial basis functions, multi-variable functional interpolation and adaptive networks, RSRE memorandum No. 4148, 1988.

[34] Zs. J. Viharos, K. B. Kis, "Diagnostics of wind turbines based on incomplete sensor data”, IMEKO World Congress Technical Diagnostics, Republic of Korea, 2012.

[35] L. A. Zadeh, "Fuzzy Sets”, Information and Control, vol. 8, pp. 338-353, 1965.

[36] J. Vieira, F. M. Dias, A. Mota, "Neuro-Fuzzy Systems: A Survey”, 5th WSEAS NNA International Conference, 2004.

[37] E. H. Mamdani, S. Assilian, “An experiment in linguistic synthesis with a fuzzy logic controller", International Journal of ManMachine Studies, vol. 7: (1), pp. 1-13, 1975.

[38] T. Takagi, M. Sugeno, "Fuzzy identification of systems and its applications to modeling and control”, IEEE Trans, on Systems, Man and Cybernetics, vol. 15, pp. 116-132, 1985.

[39] R. Jang, “Fuzzy Modeling Using Generalized Neural Networks and Kalman Filter Algorithm”, Proc. Ninth Nat. Conf. Artificial Intell., pp. 762-767, 1991.

[40] R. Jang, “ANFIS: Adaptive-Network-Based Fuzzy Inference System”, IEEE Transactions on systems, man, and cybernetics, vol. 23: (3), pp. 665-685, 1993.

[41] T. C. Lin, C. S. Lee, "Neural Network Based Fuzzy Logic Control and Decision System”, IEEE Transactions on Computers, vol. 40: (12), pp. 1320-1336, 1991.

[42] H. R. Berenji, P. Khedkar, "Learning and Tuning Fuzzy Logic Controllers Through Reinforcements”, IEEE Transactions on Neural Networks, vol. 3, pp. 724-740, 1992.

[43] D. Nauck, R. Kruse, "NEFCON-I: An XWindow Based Simulator for Neural Fuzzy Controllers", Proc. IEEE Int. Conf. Neural Networks, 1994.

[44] D. Nauck, R. Kruse, "NEFCLASS - A NeuroFuzzy Approach For The Classification Of Data”, Applied Computing Proc. of the 1995 ACM Symposium on Applied Computing, pp. 461-465, 1995.

[45] D. Nuck, R. Kurse, "Neuro-fuzzy systems for function approximation”, Fuzzy Sets and Systems, vol. 101, pp. 261-271, 1999.

[46] F. C. Juang, T. C. Lin, “An On-Line Self Constructing Neural Fuzzy Inference Network and its applications", IEEE Transactions on Fuzzy Systems, vol. 6, pp. 12-32, 1998. 
[47] N. Kasabov, Q. Song, Dynamic Evolving Fuzzy Neural Networks with 'm-out-of-n' Activation Nodes for On-Line Adaptive Systems, Technical Report TR99/04, 1999.

[48] N. Kasabov, "Adaptable Connectionist Production Systems”, Neurocomputing, vol. 13: (2-4), pp. 95-117, 1996.

[49] N. Kasabov, "Evolving Fuzzy Neural Networks - Algorithms, Applications and Biological Motivation”, Methodologies for the Conception, Design and Application of Soft Computing, pp. 271-274, 1998. 